\title{
Bayesian Inference Based on Partial Monitoring of Components with Applications to Preventive System Maintenance
}

\author{
JØRUND GÅSEMYR \\ Department of Mathematics, University of Oslo \\ BENT NATVIG \\ Department of Mathematics, University of Oslo
}

\begin{abstract}
Consider a binary, monotone system of $n$ independent components having absolutely continuous lifetime distributions. The assessment of the parameter vector, $\boldsymbol{\theta}$, of these distributions and hence of the reliability of the system is often difficult due to scarcity of data. It is therefore important to make use of all information in an efficient way. For instance, prior knowledge is often of importance and can indeed conveniently be incorporated by the Bayesian approach. It may also be important to continuously extract information from a system currently in operation. This may be useful both for decisions concerning the system in operation as well as for decisions improving the components or changing the design of similar new systems. As in Meilijson (1994) lifemonitoring of some components and conditional lifemonitoring of some others is considered. In addition to data arising from this monitoring scheme, so-called autopsy data are observed, if not censored. The probabilistic structure underlying this kind of data is described and a basic likelihood formula is arrived at. A thorough discussion of an important aspect of this probabilistic structure, the inspection strategy, is given. Based on a version of this strategy a procedure for preventive system maintenance is developed and an application to a network system presented. All the way a Bayesian approach to estimation of $\boldsymbol{\theta}$ is applied. For the case of exponentially distributed component lifetimes it is shown that the weighted sum of products of generalized gamma distributions, as introduced in Gåsemyr \& Natvig (1998), is the conjugate prior for $\boldsymbol{\theta}$.
\end{abstract}

Key words: Marked point process; likelihood function; censoring; autopsy data; inspection function; component replacement; weighted sum of products of generalized gamma distributions. 


\section{Introduction}

Consider a binary, monotone system $(E, \phi)$, where $E=\{1, \ldots, n\}$ is the set of components and $\phi$ is the structure function describing the state of the system in terms of the binary states of the components. The system may be a technological one, or a human being. We assume the components to be independent with absolutely continuous lifetime distributions. Denote the lifetime of the system by $T$ and the lifetime of the $i$ th component by $T_{i}$, with distribution function $F_{i}(t)$, survival function $\bar{F}_{i}(t)=1-F_{i}(t)$, p.d.f. $f_{i}(t)$ and failure rate $\lambda_{i}(t)=f_{i}(t) / \bar{F}_{i}(t), i \in E$. Introduce $\overline{\boldsymbol{F}}(t)=\left(\bar{F}_{1}(t), \ldots, \bar{F}_{n}(t)\right)$. The state of the $i$ th component at time $t$ is denoted $X_{i}(t)$ and we have $X_{i}(t)=I\left(T_{i}>t\right)$, $i \in E$. Let $\boldsymbol{X}(t)=\left(X_{1}(t), \ldots, X_{n}(t)\right)$. We then have $\phi(\boldsymbol{X}(t))=I(T>t)$. The reliability function, $h(\overline{\boldsymbol{F}}(t))$, of the system is given by $h(\overline{\boldsymbol{F}}(t))=E \phi(\boldsymbol{X}(t))=P(T>t)$.

The assessment of the parameter vector $\boldsymbol{\theta}$, of $\overline{\boldsymbol{F}}(t)$ and hence of the reliability function of the system is often difficult due to scarcity of data. It is therefore important to make use of all information in an efficient way. For instance, prior knowledge is often of importance and can indeed conveniently be incorporated by the Bayesian approach. It may also be important to continuously extract information from a system currently in operation. This may be useful both for decisions concerning the system in operation as well as for decisions improving the components or changing the design of similar new systems.

The Bayesian inference on $\boldsymbol{\theta}$ is based on computing the posterior distribution $\pi(\boldsymbol{\theta} \mid \boldsymbol{D}([0, t]))$, where $\boldsymbol{D}([0, t])$ denotes data arising from observing the system in the interval $[0, t]$. From Bayes theorem we have

$$
\pi(\boldsymbol{\theta} \mid \boldsymbol{D}([0, t]))=\pi_{0}(\boldsymbol{\theta}) L(\boldsymbol{\theta} \mid \boldsymbol{D}([0, t])) / \int \pi_{0}(\boldsymbol{\theta}) L(\boldsymbol{\theta} \mid \boldsymbol{D}([0, t])) d \boldsymbol{\theta},
$$

where $\pi_{0}(\boldsymbol{\theta})$ is the prior distribution and $L(\boldsymbol{\theta} \mid \boldsymbol{D}([0, t]))$ the likelihood function. When the component state processes are only partially known, some care must be taken in the computation of (1.1), even in our case of independent component processes. Such partial knowledge may arise in practice due to financial and technological limitations.

We consider a monitoring scheme in which a subset $M=\{1, \ldots, p\}$ of the components is monitored from time 0 onwards, while the components in another subset $C=\{p+$ $1, \ldots, p+q\}$, where $1 \leq p<p+q \leq n$, are conditionally monitored, i.e. they are monitored from certain time points $\tau_{i}$ onwards, $i \in C$. These time points are called inspection times and are determined by the observed history of the system according to a specific strategy determined in advance. For any component $i$ for which $T_{i} \leq T$, the failure time is recorded if it is subject to monitoring at that time. In addition to data arising from this monitoring scheme, we assume that if the system fails the set $D$ of failed components is identified through an autopsy. Data of this form has been considered by Meilijson (1994) from the point of view of maximum likelihood estimation, and by Gåsemyr (1998), who discusses the corresponding identifiability problem.

Some more terminology is needed. A path set $P$ for the system is a set of components which ensures the functioning of the system if all components in $P$ are functioning. The set $P$ is called a minimal path set if no proper subset of $P$ is a path set. A set $K$ is a cut set for the system if the system has failed when all components in $K$ have failed. A set $A$ is a fatal set if $P(D=A)>0$ where $D=\left\{i \mid T_{i} \leq T\right\}$. We denote by $\mathcal{A}$ the set $\left\{A_{1}, \ldots, A_{m}\right\}$ of fatal sets. For a fatal set $A$ we denote by $C_{A}$ the critical set corresponding 
to $A$, i.e. $C_{A}=\left\{i \in A \mid P\left(T_{i}=T \mid D=A\right)>0\right\}$, i.e. the set of components which could be the direct cause of system failure if $D=A$. It is easy to see that $C_{A}=\{i \in A \mid A-\{i\}$ is not a cut set\}.

In Section 2 of the present paper the probabilistic structure underlying the kind of data we are considering, including a censoring mechanism, is described and a basic likelihood formula is arrived at generalizing one given in Meilijson (1981). Section 3 is devoted to a thorough discussion of an important aspect of this probabilistic structure, the inspection strategy. It is also shown that the likelihood and hence the posterior distribution simplifies when applying a certain kind of inspection plan called cause-controlling. Based on a version of this inspection plan a procedure for preventive system maintenance is developed in Section 4 and an application to a network system presented. For the case of exponentially distributed component lifetimes it is shown in Section 5 that the weighted sum of products of generalized gamma distributions, as introduced in Gåsemyr \& Natvig (1998), is the conjugate prior for $\boldsymbol{\theta}$. In Appendix 1 we prove the likelihood formulae stated in Section 2 and 4, whereas some further remarks on inspection strategies are given in Appendix 2.

\section{Basic probabilistic model and likelihood formula}

A fundamental assumption in the present paper is that the inspection times are assumed to occur immediately after the failure of a component that is currently being monitored. The inspections are assumed to take zero operational time. The lifetimes $T_{1}, \ldots, T_{n}$ along with the inspection strategy determine an underlying process which is partially unobserved due to censoring at the time of system failure. The following definitions are given inductively due to the sequential nature of the set up.

$$
Z_{0}^{*}=Z_{0}=0, \quad R_{0}=M, \quad Q_{0}=\emptyset
$$

Assume $R_{k-1} \neq \emptyset$.

$Z_{k}^{*}=\min \left\{T_{i} \mid i \in R_{k-1}\right\}=$ the $k$ th smallest lifetime among components being monitored at the time of failure

$I_{k}^{*}=i \quad$ if $Z_{k}^{*}=T_{i}$

$H_{k}=$ a subset of conditionally lifemonitored components in $C-R_{k-1} \cup Q_{k-1}$ being monitored from $Z_{k}^{*}$ onwards, determined on the basis of information that is or becomes available at $Z_{k}^{*}$ about components in $M \cup C$

$H_{k, 0}=\left\{i \in H_{k} \mid T_{i} \leq Z_{k}^{*}\right\}=$ the set of conditionally lifemonitored components being failed on inspections at $Z_{k}^{*}$

$H_{k, 1}=\left\{i \in H_{k} \mid T_{i}>Z_{k}^{*}\right\}=$ the set of conditionally lifemonitored components being functioning on inspections at $Z_{k}^{*}$

$R_{k}=\left(R_{k-1}-I_{k}^{*}\right) \cup H_{k, 1}=$ the set of lifemonitored and conditionally lifemonitored components being at risk (monitored and alive) just after $Z_{k}^{*}$

$Q_{k}=\left(Q_{k-1} \cup I_{k}^{*}\right) \cup H_{k, 0}=$ the set of lifemonitored and conditionally

lifemonitored components which just after $Z_{k}^{*}$ are known to have failed 
For $i \in C$

$$
\tau_{i}^{*}= \begin{cases}Z_{k}^{*} & \text { if } i \in H_{k} \text { for some } k \\ \infty & \text { otherwise }\end{cases}
$$

The observed data are determined by this underlying process through the following equations

$$
\begin{aligned}
& Z_{k}=Z_{k}^{*} \wedge T \\
& I_{k}= \begin{cases}I_{k}^{*} & \text { if } Z_{k}=Z_{k}^{*} \\
0 & \text { otherwise }\end{cases} \\
& J_{k}= \begin{cases}0 & \text { if } Z_{k}<T \\
j & \text { if }\left(Z_{k}=T\right) \cap\left(D=A_{j}\right), j \in\{1, \ldots, m\}\end{cases} \\
& K^{\prime}=\min \left\{k \mid Z_{k}=T\right\} \\
& \tau_{i}= \begin{cases}0 & i \in M \\
\tau_{i}^{*} \wedge T & i \in C\end{cases} \\
& I=I_{K^{\prime}} \quad J=J_{K^{\prime}}
\end{aligned}
$$

If $R_{k-1}=\emptyset$, define

$$
Z_{k}^{*}=\infty, \quad H_{k}=\emptyset, \quad R_{k}=R_{k-1}=\emptyset, \quad Q_{k}=Q_{k-1}
$$

It is convenient to summarize the information obtained from the inspections immediately after $Z_{k}^{*}$ as a vector $\boldsymbol{Y}_{k}^{*}=\left(Y_{k, p+1}^{*}, \ldots, Y_{k, p+q}^{*}\right) \in\{-1,0,1\}^{q}$ defined by

$$
\boldsymbol{Y}_{k}^{*}=\left(\mathbf{1}_{H_{k, 1}}, \mathbf{0}_{H_{k, 0}},-\mathbf{1}\right),
$$

where the coordinates are 1 in the set $H_{k, 1}, 0$ in the set $H_{k, 0}$ and -1 otherwise. $H_{k}$, $H_{k, 0}$ and $H_{k, 1}$ can be recovered from $\boldsymbol{Y}_{k}^{*}$ by means of the functions $G, G_{0}$, and $G_{1}$ from $\{-1,0,1\}^{q}$ into the set of subsets of $C$ defined by

$$
\begin{aligned}
G(\boldsymbol{y}) & =\left\{i \in C \mid y_{i} \neq-1\right\} \\
G_{0}(\boldsymbol{y}) & =\left\{i \in C \mid y_{i}=0\right\} \\
G_{1}(\boldsymbol{y}) & =\left\{i \in C \mid y_{i}=1\right\} .
\end{aligned}
$$

Also define

$$
\boldsymbol{Y}_{k}= \begin{cases}\boldsymbol{Y}_{k}^{*} & \text { if } k<K^{\prime} \\ -\mathbf{1} & \text { if } k=K^{\prime}\end{cases}
$$

reflecting that there is no additional inspection after the failure of the system since by that time the autopsy data $(T, D)$ are known.

Example 2.1 To illustrate the concepts consider a parallel system with set of components $E=\{1,2,3\}, M=\{1\}$ and $C=\{2,3\}$. Assume that at the failure of component 1 , component 2 is inspected, and if it has failed component 3 is also inspected. Otherwise, component 3 is inspected at $T_{2}$. Then obviously $\tau_{1}=0, \tau_{2}=T_{1}, \tau_{3}=T_{1} \vee T_{2}$. The possible flow of events is given in Table 1. 
Table 1. Possible flow of events in a parallel system of three components

\begin{tabular}{l|c|c|c|c|c|c|c|c|}
\hline Ordering of lifetimes & $I_{1}$ & $\boldsymbol{Y}_{1}$ & $I_{2}$ & $\boldsymbol{Y}_{2}$ & $I_{3}$ & $\boldsymbol{Y}_{3}$ & $K^{\prime}$ & $\boldsymbol{Y}_{K^{\prime}}^{*}$ \\
\hline$T_{1}<T_{2}<T_{3}$ & 1 & $(1,-1)$ & 2 & $(-1,1)$ & 3 & $(-1,-1)$ & 3 & $(-1,-1)$ \\
\hline$T_{1}<T_{2}, T_{3}<T_{2}$ & 1 & $(1,-1)$ & 2 & $(-1,-1)$ & - & - & 2 & $(-1,0)$ \\
\hline$T_{2}<T_{1}<T_{3}$ & 1 & $(0,1)$ & 3 & $(-1,-1)$ & - & - & 2 & $(-1,-1)$ \\
\hline$T_{2}<T_{1}, T_{3}<T_{1}$ & 1 & $(-1,-1)$ & - & - & - & - & 1 & $(0,0)$ \\
\hline
\end{tabular}

We denote by $\mathcal{B}_{k}$ the observed history up till and including $Z_{k}$. Hence $\mathcal{B}_{k}$ is generated by the variables $Z_{1}, I_{1}, \boldsymbol{Y}_{1}, J_{1}, \ldots, Z_{k}, I_{k}, \boldsymbol{Y}_{k}, J_{k}$. The information about the system obtained at $Z_{k}$ is summarized as the vector $\left(I_{k}, \boldsymbol{Y}_{k}, J_{k}\right)$, which can be considered as the mark at $Z_{k}$ for a marked point process with $Z_{1}, \ldots, Z_{K^{\prime}}$ as the successive occurrence times. We denote the corresponding intensities by $\rho_{i, \boldsymbol{y}, j}\left(t ; Z_{1}, I_{1}, \boldsymbol{Y}_{1}, \ldots, Z_{k}, I_{k}, \boldsymbol{Y}_{k}\right), Z_{k}<t \leq Z_{k+1}$. A precise definition is given in Lemma A1.1 of Appendix 1. For $k_{0}<K^{\prime}$ we may then write the likelihood for $\boldsymbol{\theta}$ based on $\mathcal{B}_{k_{0}}$ as

$$
\begin{aligned}
& L\left(\boldsymbol{\theta} \mid \mathcal{B}_{k_{0}}\right)= \\
& \quad \prod_{k=0}^{k_{0}-1}\left\{\left[P\left(Z_{k+1}>t \mid \mathcal{B}_{k}\right)\right]_{t=Z_{k+1}} \rho_{I_{k+1}, Y_{k+1}, J_{k+1}}\left(Z_{k+1} ; Z_{1}, I_{1}, \boldsymbol{Y}_{1}, \ldots, Z_{k}, I_{k}, \boldsymbol{Y}_{k}\right)\right\} .
\end{aligned}
$$

From this equation an explicit expression for the likelihood can be calculated. First, however, we must consider the stochastic behaviour of the variables $\boldsymbol{Y}_{k+1}$. Note that if $\cup_{r=1}^{k}\left[\left\{I_{r}^{*}\right\} \cup H_{r, 0}\right] \cup\left\{I_{k+1}^{*}\right\}$ is not a cut set, the system may or may not be down at $Z_{k+1}^{*}$. Hence the inspection strategy must be based on a scheme to be followed if the system is still alive, i.e. if $T>Z_{k+1}^{*}$ but which is not effectuated if the system is down. This scheme may depend on all available information on the components at $Z_{k+1}^{*}$. Accordingly, we define $\mathcal{E}_{k}$ as the subhistory of $\mathcal{B}_{k}$ containing the same information as $\mathcal{B}_{k}$ except for information on the state of the system, i.e. whether $T>Z_{k}^{*}$ or not. Thus $\mathcal{E}_{k}$ is generated by the variables $Z_{1}^{*}, I_{1}^{*}, \boldsymbol{Y}_{1}^{*}, \ldots, Z_{k}^{*}, I_{k}^{*}, \boldsymbol{Y}_{k}^{*}$. Given $\mathcal{E}_{k}, Z_{k+1}^{*}$ and $I_{k+1}^{*}$, the available information on the states of the components at $Z_{k+1}^{*}$, only a certain subset $\mathcal{Y}_{k+1} \subseteq\{-1,0,1\}^{q}$ of values for $Y_{k+1}^{*}$ is possible as a consequence of the inspection scheme at $Z_{k+1}^{*}$. For instance, Table 1 shows that in Example 2.1 the only possibilites for $\boldsymbol{Y}_{1}^{*}$ are $(1,-1),(0,1)$ and $(0,0)$.

A convenient sufficient condition to establish our likelihood formula, turns out to be given by the last equality to follow

$$
\begin{array}{rl}
\lim _{d t \rightarrow 0} & P\left[\boldsymbol{Y}_{k+1}^{*}=\boldsymbol{y} \mid \mathcal{E}_{k} \cap\left(t<Z_{k+1}^{*} \leq t+d t\right) \cap\left(I_{k+1}^{*}=i\right)\right] \\
& =\lim _{d t \rightarrow 0} P\left[\boldsymbol{Y}_{k+1}^{*}=\boldsymbol{y} \mid \mathcal{E}_{k} \cap\left(t<T_{i} \leq t+d t\right) \cap\left(\bigcap_{\ell \in R_{k}-\{i\}}\left(T_{\ell}>T_{i}\right)\right)\right] \\
& =\prod_{\ell \in G_{0}(\boldsymbol{y})} F_{\ell}(t) \prod_{\ell \in G_{1}(\boldsymbol{y})} \bar{F}_{\ell}(t) \quad \text { for } \boldsymbol{y} \in \mathcal{Y}_{k+1},
\end{array}
$$

and 0 otherwise. Note that unlike $\mathcal{B}_{k}$, the subhistory $\mathcal{E}_{k}$, does not contain any probabilistic information on the states of the components in $C-\left(R_{k} \cup Q_{k}\right)$, i.e. these components are conditionally independent with conditional lifetime distributions equal to the original distributions. Hence, (2.3) is a very natural condition. Basically, (2.3) expresses a certain stability in the inspection strategy, excluding measure theoretically pathological cases. Some further comments on this are given in Appendix 2. 
In order to include a censoring mechanism, suppose $V>0$ is a censoring time, either fixed in advance or a random variable, being independent of $T_{i}, i=1, \ldots, n$, and not depending on our parameter vector $\boldsymbol{\theta}$. The system is observed in $[0, S]$, where $S=T \wedge V$. Introduce

$$
K=\max \left\{k \mid Z_{k}<S\right\} \quad N=\bigcup_{k=1}^{K} H_{k} \quad N_{i}=\bigcup_{k=1}^{K} H_{k, i}, \quad i=0,1
$$

$N, N_{0}$ and $N_{1}$ are the sets of components that respectively are inspected, are failed on inspections and are functioning on inspections, up till and including the time $Z_{K}$.

Our basic likelihood formula is given in Theorem 2.1, which is proved in Appendix 1.

Theorem 2.1 Let $\delta_{i}=I\left(\tau_{i}<T_{i}<S\right), i \in M \cup C$. Then the complete likelihood function for our parameter vector, $\boldsymbol{\theta}$, is given by

$$
\begin{aligned}
L(\boldsymbol{\theta}) & =\prod_{i \in M \cup C}\left(\lambda_{i}\left(T_{i}\right)\right)^{\delta_{i}} \prod_{i \in M \cup N_{1}} \bar{F}_{i}\left(T_{i} \wedge S\right) \prod_{i \in N_{0}} F_{i}\left(\tau_{i}\right) \\
& \times\left\{I ( V > S ) \left[I(I \neq 0) \lambda_{I}(S) \prod_{\ell \in A_{J}-(M \cup N)} F_{\ell}(S) \prod_{\ell \in A_{J}^{c}-(M \cup N)} \bar{F}_{\ell}(S)\right.\right. \\
& \left.+I(I=0) \sum_{i \in C_{A_{J}}-(M \cup N)} \lambda_{i}(S) \prod_{\ell \in A_{J}-(M \cup N \cup\{i\})} F_{\ell}(S) \prod_{\ell \in\left(A_{J}^{c} \cup\{i\}\right)-(M \cup N)} \bar{F}_{\ell}(S)\right] \\
& \left.+I(V=S) h\left(\mathbf{1}_{R_{K}}, \mathbf{0}_{Q_{K}}, \overline{\boldsymbol{F}}(S)\right)\right\} .
\end{aligned}
$$

Note that the first line in the expression for $L(\boldsymbol{\theta})$ represents the full likelihood function up till just before system failure or censoring. The factor multiplied by $I(V>S) I(I \neq 0)$ is the contribution to $L(\boldsymbol{\theta})$ from a system failure due to the failure of a currently monitored component. Similarly, the factor multiplied by $I(V>S) I(I=0)$ is the contribution from a system failure due to the failure of a component that is not currently being monitored. Finally, the factor multiplied by $I(V=S)$ is the conditional survival probability of the system up till censoring. By setting $M=C=\emptyset, V=\infty$ noting that we then always have $I=0, L(\boldsymbol{\theta})$ reduces to the one given in Meilijson (1981).

\section{$3 \quad$ Inspection strategies}

As a background for discussing inspection strategies in general we start out from a slightly extended Example 2.1.

Example 3.1 Consider a parallel system with $E=\{1,2,3\}, M=\{1\}$. By symmetry we may assume $C=\{2\}$ or $C=\{2,3\}$. Let $\tau_{1}=0, \tau_{2}=Z_{1}^{*}=T_{1}$. The possible inspection strategies at $Z_{1}^{*}$ can be characterized by the corresponding possible sets $\mathcal{Y}_{1}$. All possibilities are given in Table 2 .

Case iii) is just the strategy of Example 2.1. Since after $T_{1} \vee T_{2}$ the system state equals the state of component 3 , the cases i) and iii) as well as the cases ii) and iv) give the same information.

We see from this example that two different inspection strategies may give the same information even if one of them has fewer monitored components (cases i) and iii)) or 
Table 2. All possible inspection strategies at $Z_{1}^{*}$ for a parallel system of three components

\begin{tabular}{|l|c|c|c|c|}
\hline Case number & i) & ii $)$ & iii & iv $)$ \\
\hline$C$ & $\{2\}$ & $\{2,3\}$ & $\{2,3\}$ & $\{2,3\}$ \\
\hline $\mathcal{Y}_{1}$ & 1,0 & $(1,1),(1,0),(0,1),(0,0)$ & $(1,-1),(0,1),(0,0)$ & $(1,1),(1,0),(0,-1)$ \\
\hline
\end{tabular}

different inspection times (cases ii) and iv)). In general consider a component, say $i$ (being component 3 above), known to be critical for the system at $Z_{k}^{*}$. Then for the first time $Q_{k} \cup\{i\}$ is a cut set, while $Q_{k}$ is not. Even if $i \notin C$ (case i)) it may be considered as conditionally monitored from $Z_{k}^{*}$ onwards (as in case iii)). The reason is that if component $i$ then later fails along with the system, this is revealed in the autopsy data, and hence we get the same information as if $i \in C$.

Now consider the case $i \in C$. Component $i$ may then be considered as conditionally monitored from $Z_{k}^{*}$ onwards (as in case ii)) even if $\tau_{i}^{*}>Z_{k}^{*}$ (as in case iv)). Again we get the same information since a failure of component $i$ is anyway revealed in the autopsy data.

It follows from these considerations that it is unnecessary to physically inspect or monitor a component which is known to be critical for the system if the purpose of the inspection strategy is to update the prior distributions of $\boldsymbol{\theta}$ with operational data. However, the inspection strategy may serve other purposes, e.g. recording performance level, load or aging, and may therefore be justified.

The inspection strategy may change with time. For instance, one may in Example 3.1 switch between cases i) and ii) at fixed time points. This means that depending on $T_{1}$, but independently of the states of components 2 and 3, one inspects either only component 2 or both components 2 and 3 . A way of constructing inspection strategies which generalize such a scheme is to specify for each possible history $\mathcal{E}_{k}$ and each $i \in R_{k}$, a right continuous and hence necessarily piecewise constant function $H_{i}^{\mathcal{E}_{k}}(t), t>Z_{k}^{*}$, into the set of subsets of $C-\left(R_{k} \cup Q_{k}\right)$, and then define

$$
H_{k+1}=H_{I_{k+1}^{*}}^{\mathcal{E}_{k}}\left(Z_{k+1}^{*}\right)
$$

It is verified in Appendix 2 that (2.3) is now satisfied. Specifying functions $H_{i}^{\mathcal{E}_{k}}(t)$ for all possible histories might seem like an enormous task, but in practice only a few essential features of the information in $\mathcal{E}_{k}$ may be needed. For instance, we may let $H_{k+1}$ depend on $\mathcal{E}_{k}, I_{k+1}^{*}$ and $Z_{k+1}^{*}$ only through $\left(R_{k}-\left\{I_{k+1}^{*}\right\}, Q_{k} \cup\left\{I_{k+1}^{*}\right\}\right)$. This gives an inspection plan of type 1, as defined in Gåsemyr (1998). Accordingly, we call a strategy based on the more general (3.1) a history dependent inspection plan of type 1.

As a generalization of strategy iii) of Table 2, a more flexible strategy can be defined by allowing $H_{k+1}$ to depend more extensively on information that becomes available at $Z_{k+1}^{*}$. We then let $H_{k+1}$ be determined through an iterative procedure involving a function $H_{i}^{\mathcal{E}_{k}}(t, R, Q)$ into $\{C-R \cup Q\} \cup\{\emptyset\}$, being piecewise constant and right continuous in $t>Z_{k}^{*}$. Here $i \in R_{k}$, whereas $R$ and $Q$ are disjoint subsets of $C$ with $R_{k}-\{i\} \subseteq R$ and $Q_{k} \cup\{i\} \subseteq Q$.

$H_{k+1}$ is then the result of repeated applications of $H_{I_{k+1}^{*}}^{\mathcal{E}_{k}}\left(Z_{k+1}^{*}, R, Q\right)$ with $(R, Q)=$ $\left(R_{k}-\left\{I_{k+1}^{*}\right\}, Q_{k} \cup\left\{I_{k+1}^{*}\right\}\right)$ as initial values. For each iteration, a new component is selected for inspection and afterwards the pair $(R, Q)$ is updated by adding the component to $R$ 
if it is functioning and to $Q$ if not. This procedure is stopped when for the first time $H_{I_{k+1}^{*}}^{\mathcal{E}_{k}}\left(Z_{k+1}^{*}, R, Q\right)=\emptyset$. We assume the procedure takes zero operational time. It is again verified in Appendix 2 that (2.3) is satisfied.

If $H_{i}^{\mathcal{E}_{k}}(t, R, Q)=H(R, Q)$ depends on the history only through the pair $(R, Q)$ of components known to respectively be at risk and to have failed, $H$ is called an inspection function and the strategy is an inspection plan of type 2 according to the terminology in Gåsemyr (1998). In case iii) of Table $3.1 H(\emptyset,\{1\})=\{2\}$ and $H(\emptyset,\{1,2\})=\{3\}$.

The design of inspection plans is an important problem, which, however, will not be discussed in depth in the present paper. One purpose may be to acquire operational information in order to predict the future performance of the system at hand. This is illustrated in the following example considering a history dependent inspection plan of type 2 .

Example 3.2 Assume due to scarcity of financial resources, technical equipment or personnel, it is only possible to monitor a fixed number of components at a time. Whenever a currently monitored component fails, exactly one new component is added to the risk set. To decide which component to start monitoring at $Z_{k+1}^{*}$, we order the components in $C-R_{k} \cup Q_{k}$ according to the size of the quantity

$$
M_{i}(k+1)=E\left[T \mid \mathcal{B}_{k+1} \cap\left(X_{i}\left(Z_{k+1}^{*}\right)=1\right)\right]-E\left[T \mid \mathcal{B}_{k+1} \cap\left(X_{i}\left(Z_{k+1}^{*}\right)=0\right)\right] .
$$

The components are inspected in decreasing order of $M_{i}(k+1)$ until a functioning one is found. This component is then included in the risk set. The motivation is that a component with larger $M_{i}(k+1)$ carries more information about the expected system lifetime. This quantity is very much linked to the Natvig measure of importance of components, see Natvig (1985).

For $\delta=0,1$ we have

$$
\begin{aligned}
P[T & \left.>t \mid \mathcal{B}_{k+1} \cap\left(X_{i}\left(Z_{k+1}^{*}\right)=\delta\right)\right] \\
& =\int P\left[T>t \mid \mathcal{B}_{k+1} \cap\left(X_{i}\left(Z_{k+1}^{*}\right)=\delta\right), \boldsymbol{\theta}\right] \pi\left(\boldsymbol{\theta} \mid \mathcal{B}_{k+1} \cap\left(X_{i}\left(Z_{k+1}^{*}\right)=\delta\right)\right) d \boldsymbol{\theta} \\
& \propto \int P\left[T>t \mid \mathcal{B}_{k+1} \cap\left(X_{i}\left(Z_{k+1}^{*}\right)=\delta\right), \boldsymbol{\theta}\right) L\left(\boldsymbol{\theta} \mid \mathcal{B}_{k+1} \cap\left(X_{i}\left(Z_{k+1}^{*}\right)=\delta\right)\right) \pi_{0}(\boldsymbol{\theta}) d \boldsymbol{\theta},
\end{aligned}
$$

where $\pi\left(\boldsymbol{\theta} \mid \mathcal{B}_{k+1} \cap\left(X_{i}\left(Z_{k+1}^{*}\right)=\delta\right)\right)$ is the posterior distribution of $\boldsymbol{\theta} . M_{i}(k+1)$ is determined by (3.3) since

$$
M_{i}(k+1)=\int_{0}^{\infty}\left\{P\left[T>t \mid \mathcal{B}_{k+1} \cap\left(X_{i}\left(Z_{k+1}^{*}\right)=1\right)\right]-P\left[T>t \mid \mathcal{B}_{k+1} \cap\left(X_{i}\left(Z_{k+1}^{*}\right)=0\right)\right]\right\} d t .
$$

Hence we see that the current updating of the information on $\boldsymbol{\theta}$ is not just a consequence of our monitoring scheme, but is in fact an integrated part of the scheme. The computation of $M_{i}(k+1)$ must in most cases be carried through by simulation. One starts by generating a sample from the posterior distribution of $\boldsymbol{\theta}$ by for instance rejection sampling, and then simulates the process $\boldsymbol{X}(t)$ for given $\boldsymbol{\theta}$.

We now consider a version of an inspection plan of type 2, introduced in Gåsemyr (1998) and called cause-controlling, which is applied in the next section when considering preventive system maintenance. An inspection plan is cause-controlling if for $k=0,1, \ldots$, 
the risk set $R_{k+1}$, arising from the whole inspection immediately after $Z_{k+1}^{*}$, is always a path set for the system if possible. This can be achieved by using an inspection function $H$ satisfying $H(R, Q) \neq \emptyset$ if $R$ is not a path set. Suppose in addition that $M \cup C=E$ and that $R_{0}=M$ is a path set. It is then easy to see that the system failure time $T$ must coincide with the failure time of a component that is currently being monitored. Hence, the identity of the component causing system failure becomes known.

The following corollary is immediate from Theorem 2.1 since for a cause-controlling inspection plan we always have $I \neq 0$. In addition, the contribution $h\left(\mathbf{1}_{R_{K}}, \mathbf{0}_{Q_{K}}, \overline{\boldsymbol{F}}(S)\right)$ equals 1 since $R_{K}$ is now a path set.

Corollary 3.1 For the case of a cause-controlling inspection plan the complete likelihood function for our parameter vector, $\boldsymbol{\theta}$, reduces to

$$
\begin{aligned}
L(\boldsymbol{\theta}) & =\prod_{i \in M \cup C}\left(\lambda_{i}\left(T_{i}\right)\right)^{\delta_{i}} \prod_{i \in M \cup N_{1}} \bar{F}_{i}\left(T_{i} \wedge S\right) \prod_{i \in N_{0}} F_{i}\left(\tau_{i}\right) \\
& \times\left\{I(V>S) \lambda_{I}(S) \prod_{\ell \in A_{J}-(M \cup N)} F_{\ell}(S) \prod_{\ell \in A_{J}^{c}-(M \cup N)} \bar{F}_{\ell}(S)+I(V=S)\right\}
\end{aligned}
$$

We conclude this section by giving another corollary following immediately from (1.1) and Corollary 3.1

Corollary 3.2 Let $\boldsymbol{\theta}=\left(\boldsymbol{\theta}_{1}, \ldots, \boldsymbol{\theta}_{u}\right), u \leq n$. Suppose that for each $i \in E$ there exists a unique $r_{i} \in\{1, \ldots, u\}$ such that $F_{i}$ depends only on $\boldsymbol{\theta}_{r_{i}}$. If $\boldsymbol{\theta}_{1}, \ldots, \boldsymbol{\theta}_{u}$ are independent $\grave{a}$ priori and the likelihood function is based on data from a system monitored according to a cause-controlling inspection plan, then $\boldsymbol{\theta}_{1}, \ldots, \boldsymbol{\theta}_{u}$ are independent à posteriori as well.

\section{An application to preventive system maintenance}

In this section we consider preventive system maintenance where components are replaced according to a specific strategy. We have to take into account that it is costly to intervene in system operation. Hence, it is desirable to postpone replacement of failed components as long as possible in order to replace several components at a time. On the other hand, it is obviously important to avoid a system failure. As a compromise we assume that components are replaced as soon as system weakening has reached a certain level; i.e. when $\psi(\boldsymbol{X}(t))$ jumps to zero, where $\psi$ is a binary, monotone structure function such that $\psi(\boldsymbol{X}(t)) \leq \phi((\boldsymbol{X}(t))$. At this time a total inspection of the components is carried through and all failed components are replaced, while the others are not affected. We assume this procedure takes zero operational time. Afterwards, the replaced components are assumed to have the same lifetime distributions as the initial ones.

It is natural to choose $\psi$ such that when $\psi(\boldsymbol{X}(t))$ jumps to zero, at least one additional component must fail for $\phi(\boldsymbol{X}(t))$ to jump to zero. If for instance $\phi$ is a $k$-out-of- $n$ system, we can choose $\psi$ as a $(k+1)$-out-of- $n$ system. Let now $\phi$ be the structure function of an undirected network system. How does one in general find a corresponding structure function $\psi$, if it exists? The problem is solved if given the minimal path sets $P_{1}, \ldots, P_{p}$ corresponding to $\phi$, one can find the minimal path sets corresponding to a $\psi$. By Menger's 
theorem, see Bondy \& Murty (1976), it can be shown formally that every path set for $\psi$ must contain at least two disjoint minimal path sets for $\phi$, which is intuitively obvious. Hence the set of minimal path sets for $\psi$ can be chosen to be the minimal sets in $\left\{P_{i} \cup\right.$ $\left.P_{j} \mid P_{i} \cap P_{j}=\emptyset\right\}$. This result can be extended to directed network systems by applying the max-flow-min-cut theorem, again see Bondy \& Murty (1976). An application to an undirected network system is given in the following.

Example 4.1 Consider the network system of seven components given in Figure 1.

Figure 1. Network system of seven components

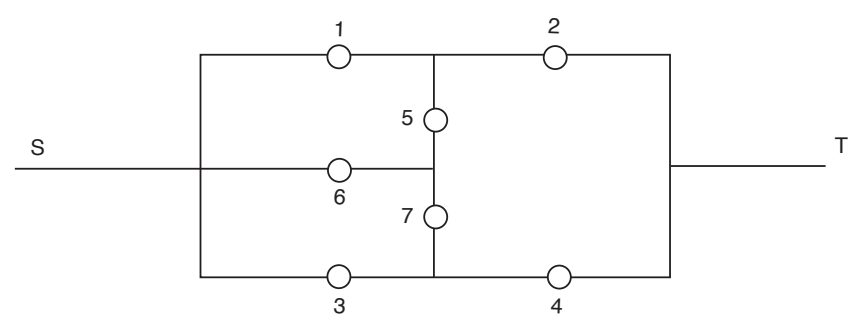

The system is working iff there is at least one connection between $\mathrm{S}$ (ource) and $\mathrm{T}$ (erminal). The minimal path sets of this system is obviously $\{1,2\},\{1,5,7,4\},\{6,5,2\}$, $\{6,7,4\},\{3,4\},\{3,7,5,2\}$ giving the minimal path sets $\{1,2,3,4\},\{1,2,4,6,7\},\{2,3,4,5,6\}$ of the corresponding $\psi$ system. This leads to

$$
\psi(\boldsymbol{x})=x_{2} x_{4}\left[x_{1} x_{3}+\left(1-x_{3}\right) x_{1} x_{6} x_{7}+\left(1-x_{1}\right) x_{3} x_{5} x_{6}\right]
$$

We denote by $T_{i, \ell}$ and $S_{i, \ell}$ respectively the time for the $\ell$ th failure and replacement of the $i$ th component, $i \in E, \ell=1,2, \ldots$ The successive times of preventive system maintenance are denoted $T^{1}, T^{2}, \ldots$, and the interval $\left(T^{r-1}, T^{r}\right]$ is called the $r$ th operational period of the system, $r=1,2, \ldots$ Here $T^{0}=0$. Formally, these variables are related as follows. Let

$$
\begin{aligned}
& X_{i, 1}(t)=I\left(T_{i, 1}>t\right), \quad i \in E, \quad t>0 \\
& \boldsymbol{X}_{1}(t)=\left(X_{1,1}(t), \ldots, X_{n, 1}(t)\right) \\
& T^{1}=\inf \left\{t>0 \mid \psi\left(\boldsymbol{X}_{1}(t)\right)=0\right\} \\
& X_{i, 2}(t)=I\left(T_{i, 1}>t\right)+I\left(T_{i, 1} \leq T^{1}\right) I\left(T_{i, 2}>t\right), \quad i \in E, \quad t>T^{1} \\
& \boldsymbol{X}_{2}(t)=\left(X_{1,2}(t), \ldots, X_{n, 2}(t)\right) \\
& T^{2}=\inf \left\{t>T^{1} \mid \psi\left(\boldsymbol{X}_{2}(t)\right)=0\right\} \\
& \quad \quad \quad \\
& \quad \quad+I\left(T_{i, r-1} \leq T^{r-1}\right) I\left(T_{i, r}>t\right), \quad i \in E, \quad t>T^{r-1} \\
& X_{i, r}(t)=I\left(T_{i, 1}>t\right)+I\left(T_{i, 1} \leq T^{r-1}\right) I\left(T_{i, 2}>t\right)+\cdots \\
& \boldsymbol{X}_{r}(t)=\left(X_{1, r}(t), \ldots, X_{n, r}(t)\right) \\
& T^{r}=\inf \left\{t>T^{r-1} \mid \psi\left(\boldsymbol{X}_{r}(t)\right)=0\right\} \\
& S_{i, 0}=0 \\
& S_{i, \ell}=\min \left\{T^{r}, r \in\{1,2, \ldots\} \mid T_{i, \ell} \leq T^{r}\right\}, \quad \ell=1,2, \ldots
\end{aligned}
$$


The component states at time $t$ are given by

$$
\boldsymbol{X}(t)=\sum_{r=1}^{\infty} I\left(T^{r-1}<t \leq T^{r}\right) \boldsymbol{X}_{r}(t)
$$

Our distributional assumptions can formally be stated by introducing

$$
V_{i, \ell}=T_{i, \ell}-S_{i, \ell-1}, \quad i \in E, \quad \ell=1,2, \ldots
$$

Then the variables $V_{i, \ell}$ are independent and $V_{i, \ell}$ has distribution function $F_{i}(t)$.

In order to immediately register the successive times, $T^{1}, T^{2}, \ldots$, of preventive system maintenance, an inspection plan of type 2 , which is cause-controlling with respect to $\psi$, is followed. At the consecutive failure times, $Z_{k}^{*}$, of the currently monitored components, the risk set is updated by $H_{k, 1}$, the set of conditionally lifemonitored components being functioning on inspection, $k=1,2, \ldots$ If $Z_{k}^{*}=T^{r}$ for some $r=1,2, \ldots$, a total inspection of the components is carried through and all failed components are replaced, while the others are not affected. We then set $R_{k}=M$.

Example 4.1 continued To have a cause-controlling inspection plan with respect to $\psi$ it is natural to choose $M=\{1,2,3,4\}$ and $C=\{5,6,7\}$. If either component 2 or 4 fails first, the $\psi$ system fails and all failed components are replaced. If component 1 fails first, components 5 and 6 are inspected and if at least one of them has failed, the $\psi$ system has failed and all failed components are replaced. If on the other hand both components 5 and 6 have survived, they are now monitored. Then when the first of the components in $\{2,3,4,5,6\}$ fails, the $\psi$ system fails and all failed components are replaced. Completely parallel, if component 3 fails first, the above procedure is still valid by replacing components 1 and 5 with respectively 3 and 7 .

For $i \in C, r=0,1,2, \ldots$ the inspection strategy is given by inspection times $\tau_{i}^{r}$ defined as follows

$$
\tau_{i}^{r_{*}}=\min \left\{Z_{k}^{*} \mid\left(Z_{k}^{*}>T^{r}\right) \cap\left(i \in H_{k}\right)\right\} \quad \tau_{i}^{r}=\tau_{i}^{r_{*}} \wedge T^{r+1} .
$$

Let for $i \in E, \ell=1,2, \ldots$

$$
\begin{aligned}
& R_{i, \ell}= \max \left\{r \in\{1,2, \ldots\} \mid T^{r}<S_{i, \ell}\right\} \\
&= \text { the number of operational periods of the system before the one that ends } \\
& \text { with the } \ell \text { th replacement of the } i \text { th component } \\
& \tau_{i, \ell}= \begin{cases}0, & i \in M \\
\tau_{i}^{R_{i, \ell}}, & i \in C\end{cases}
\end{aligned}
$$

We want to calculate the likelihood function, $L(\boldsymbol{\theta})$, based on data from observing the system components according to the scheme described on the interval $\left[0, t_{0}\right]$, where $t_{0}$ is either a fixed time point or the result of random censoring. For $i \in E, \ell=1,2, \ldots$ define 


$$
\begin{aligned}
& \widetilde{T}_{i, 0}=0 \\
& \widetilde{T}_{i, \ell}=\quad T_{i, \ell} \vee \tau_{i, \ell}=\text { the time when the } \ell \text { th failure of the } i \text { th component is known } \\
& L_{i}=\max \left\{\ell \in\{0,1, \ldots\} \mid \widetilde{T}_{i, \ell} \leq t_{0}\right\} \\
& \quad=\quad \text { the number of known failures for the } i \text { th component before } t_{0} \\
& \delta_{i, \ell}=I\left(\tau_{i, \ell} \leq T_{i, \ell}\right) \\
& R=\max \left\{r \in\{1,2, \ldots\} \mid T^{r} \leq t_{0}\right\} \\
& \quad=\quad \text { the number of operational periods of the system completed before } t_{0} \\
& R\left(t_{0}\right)=\text { the risk set at } t_{0} .
\end{aligned}
$$

For $r=0, \ldots, R$ define $k_{r}$ by

$$
Z_{k_{r}}^{*}=T^{r}
$$

Note that since $Z_{0}^{*}=T^{0}=0$, we have $k_{0}=0$. Finally, let

$$
\begin{aligned}
G(R)= & E-\left(\bigcup_{\left\{k \geq k_{R}+1 \mid Z_{k}^{*}<t_{0}\right\}}\left(H_{k, 0} \cup\left\{I_{k}^{*}\right\}\right) \cup R\left(t_{0}\right)\right) \\
= & \text { the set of components for which no information is acquired in the interval } \\
& \left(T^{R}, t_{0}\right] .
\end{aligned}
$$

The following theorem is proved in Appendix 1.

Theorem 4.1 The complete likelihood function for our parameter vector, $\boldsymbol{\theta}$, in the component replacement model, where all failed components are replaced, is given by

$$
\begin{aligned}
L(\boldsymbol{\theta}) & =\prod_{i \in E} \prod_{\ell=1}^{L_{i}}\left[\lambda_{i}\left(T_{i, \ell}-S_{i, \ell-1}\right) \bar{F}_{i}\left(T_{i, \ell}-S_{i, \ell-1}\right)\right]^{\delta_{i, \ell}} \\
& \times\left[F_{i}\left(\tau_{i, \ell}-S_{i, \ell-1}\right)-F_{i}\left(T^{R_{i, \ell}}-S_{i, \ell-1}\right)\right]^{1-\delta_{i, \ell}} \\
& \times \prod_{i \in R\left(t_{0}\right)} \bar{F}_{i}\left(t_{0}-S_{i, L_{i}}\right) \prod_{i \in G(R)} \bar{F}_{i}\left(T^{R}-S_{i, L_{i}}\right) .
\end{aligned}
$$

Example 4.1 continued To illustrate Theorem 4.1 and the associated notation we have simulated 20 exponentially distributed lifetimes for each of the components $1, \ldots, 7$ with expectations $\theta_{i}^{-1}, i=1, \ldots, 7$ measured in hours respectively equal to 1000,2000 , $1800,1500,600,800,700$. We chose $t_{0}=10000$ hours. For $i \in M=\{1,2,3,4\}, \delta_{i, \ell}=1$ and only $T_{i, \ell}$ and $S_{i, \ell-1}$ is needed to calculate the corresponding contribution to $L(\boldsymbol{\theta})$. Based on the results of the simulations all relevant calculations to arrive at $L(\boldsymbol{\theta})$ are given in Table 3.

From Table 3 we see that $R=21, L_{1}=11 L_{2}=3, L_{3}=5, L_{4}=5, L_{5}=10$, $L_{6}=13, L_{7}=11, R\left(t_{0}\right)=M=\{1,2,3,4\}, G(R)=C=\{5,6,7\}$. Applying Theorem 4.1 straightforward calculations give

$$
\begin{aligned}
& L(\boldsymbol{\theta})=\theta_{1}^{11} e^{-9395 \theta_{1}} \theta_{2}^{3} e^{-10000 \theta_{2}} \theta_{3}^{5} e^{-9128 \theta_{3}} \theta_{4}^{5} e^{-10000 \theta_{4}} \\
& \quad \times \theta_{5}^{2} e^{-4497 \theta_{5}}\left(1-e^{-449 \theta_{5}}\right)\left(1-e^{-273 \theta_{5}}\right)\left(1-e^{-447 \theta_{5}}\right)\left(1-e^{-337 \theta_{5}}\right)\left(1-e^{-1614 \theta_{5}}\right) \\
& \quad \times\left(1-e^{-860 \theta_{5}}\right)\left(1-e^{-1223 \theta_{5}}\right)\left(1-e^{-295 \theta_{5}}\right) \\
& \quad \times \theta_{6} e^{-2904 \theta_{6}}\left(1-e^{-866 \theta_{6}}\right)\left(1-e^{-346 \theta_{6}}\right)\left(1-e^{-253 \theta_{6}}\right)\left(1-e^{-499 \theta_{6}}\right)\left(1-e^{-449 \theta_{6}}\right)\left(1-e^{-115 \theta_{6}}\right)
\end{aligned}
$$




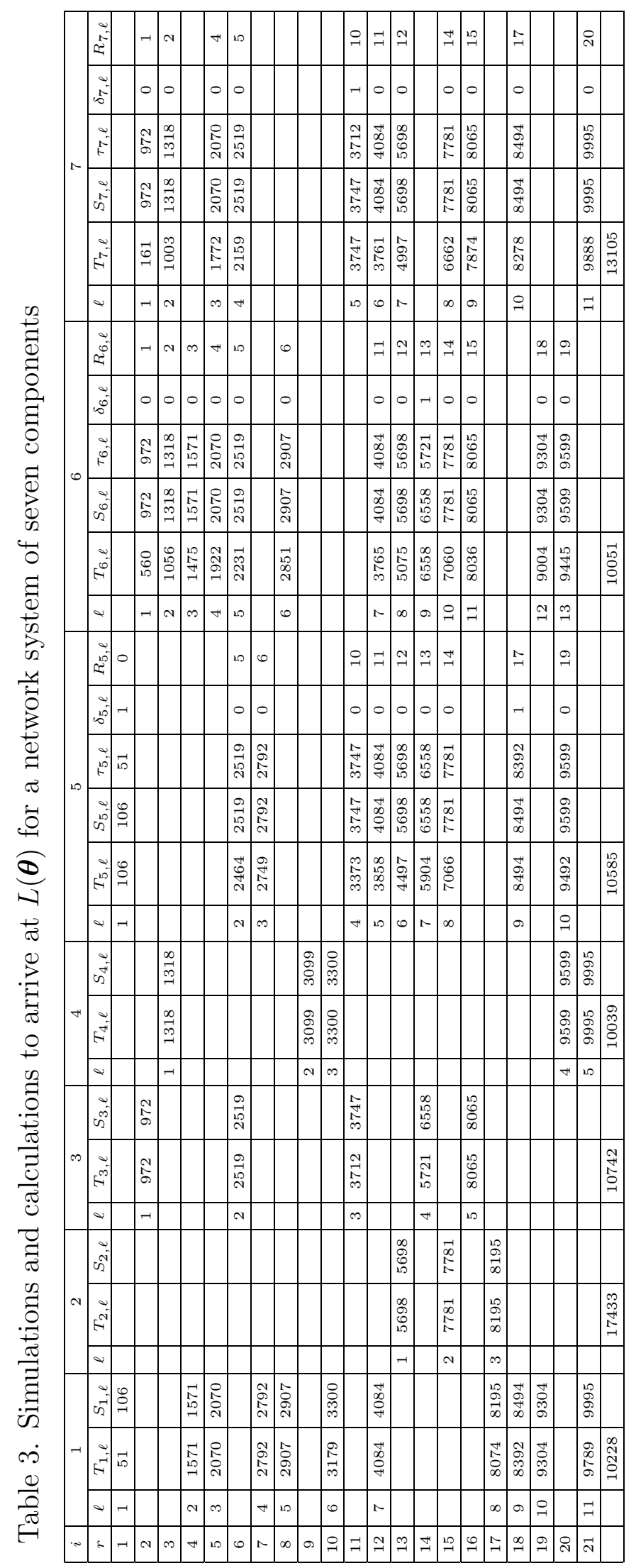




$$
\begin{aligned}
& \times\left(1-e^{-337 \theta_{6}}\right)\left(1-e^{-1614 \theta_{6}}\right)\left(1-e^{-1223 \theta_{6}}\right)\left(1-e^{-284 \theta_{6}}\right)\left(1-e^{-810 \theta_{6}}\right)\left(1-e^{-295 \theta_{6}}\right) \\
& \times \theta_{7} e^{-3682 \theta_{7}}\left(1-e^{-866 \theta_{7}}\right)\left(1-e^{-346 \theta_{7}}\right)\left(1-e^{-499 \theta_{7}}\right)\left(1-e^{-449 \theta_{7}}\right)\left(1-e^{-337 \theta_{7}}\right) \\
& \times\left(1-e^{-1614 \theta_{7}}\right)\left(1-e^{-1223 \theta_{7}}\right)\left(1-e^{-284 \theta_{7}}\right)\left(1-e^{-299 \theta_{7}}\right)\left(1-e^{-396 \theta_{7}}\right)
\end{aligned}
$$

An alternative component replacement model is obtained if at $Z_{k_{r}}^{*}, r=1, \ldots, R$ no inspection of the components is carried through at all and hence only components known to have failed are replaced. Since the system state after replacement now is at best as good as if all failed components were replaced, more components should be monitored suggesting that $M \subset R_{k_{r}}$. We set $R_{k_{r}}=\left(R_{k_{r}-1}-I_{k_{r}}^{*}\right) \cup M$. Define inductively

$$
\begin{aligned}
& S_{i, 0}=0 \\
& \tau_{i, \ell}=\min \left\{Z_{k}^{*} \mid\left(Z_{k}^{*} \geq S_{i, \ell-1}\right) \cap\left(i \in H_{k}\right)\right\} \\
& S_{i, \ell}=\min \left\{T^{r}, r \in\{1,2, \ldots\} \mid \widetilde{T}_{i, \ell} \leq T^{r}\right\} .
\end{aligned}
$$

Parallel to Theorem 4.1 we now get the following theorem, which is proved in Appendix 1.

Theorem 4.2 The complete likelihood function for our parameter vector, $\boldsymbol{\theta}$, in the component replacement model, where only components known to have failed are replaced, is given by

$$
\begin{aligned}
L(\boldsymbol{\theta})= & \prod_{i \in M \cup C} \prod_{\ell=1}^{L_{i}}\left[\lambda_{i}\left(T_{i, \ell}-S_{i, \ell-1}\right) \bar{F}_{i}\left(T_{i, \ell}-S_{i, \ell-1}\right)\right]^{\delta_{i, \ell}} \\
& \times\left[F_{i}\left(\tau_{i, \ell}-S_{i, \ell-1}\right)\right]^{1-\delta_{i, \ell}} \prod_{i \in R\left(t_{0}\right)} \bar{F}_{i}\left(t_{0}-S_{i, L_{i}}\right) .
\end{aligned}
$$

\section{Bayesian estimation of component parameters}

The posterior distribution of $\boldsymbol{\theta}$ given by (1.1) is the basis for Bayesian inference on component lifetimes. A specific parameter may for instance be estimated by the expectation in its posterior marginal distribution. When exact methods for calculating the expectation are not available, one may use Markov Chain Monte Carlo simulation to obtain approximate values. See for instance Smith \& Roberts (1993).

Now assume that the lifetime of the $i$ th component, $T_{i}$, is exponentially distributed with failure rate $\theta_{i}, i \in E$. We have $\boldsymbol{\theta}=\left(\theta_{1}, \ldots, \theta_{n}\right)$.

The following definition of the generalized gamma distribution is given in Gåsemyr \& Natvig (1998).

Definition 5.1 For positive real numbers $a, b, t_{1}, \ldots, t_{r}$ define the functions

$$
\begin{aligned}
& f(\theta ; a, b, \boldsymbol{t})=\theta^{a-1} e^{-b \theta} \prod_{i=1}^{r}\left(1-e^{-\theta t_{i}}\right), \quad \theta \geq 0 \\
& f(\theta ; a, b)=\theta^{a-1} e^{-b \theta}, \quad \theta \geq 0,
\end{aligned}
$$

where $\boldsymbol{t}=\left(t_{1}, \ldots, t_{r}\right)$. Define the normalizing constant $\gamma(a, b, \boldsymbol{t})$ by

$$
(\gamma(a, b, \boldsymbol{t}))^{-1}=\Gamma(a) \sum_{\boldsymbol{d} \in\{0,1\}^{r}}(-1)^{|\boldsymbol{d}|}(b+\boldsymbol{d} \cdot \boldsymbol{t})^{-a},
$$


where $|\boldsymbol{d}|=d_{1}+\cdots+d_{r}$. The generalized gamma distribution with parameters $a, b$ and $\boldsymbol{t}$ is then defined as the probability distribution on $[0, \infty)$ with density function given by

$$
g(\theta ; a, b, \boldsymbol{t})=\gamma(a, b, \boldsymbol{t}) f(\theta ; a, b, \boldsymbol{t}), \quad \theta \geq 0 .
$$

The ordinary gamma distribution, $g(\theta ; a, b)$, is the special case corresponding to $r=0$.

Note that conditionally on $\boldsymbol{\theta}$, the reliability function of the system can be written as

$$
h(\overline{\boldsymbol{F}}(t))=\sum_{\boldsymbol{x} \in\{0,1\}^{n}} \phi(\boldsymbol{x}) \prod_{i=1}^{n}\left(e^{-\theta_{i} t}\right)^{x_{i}}\left(1-e^{-\theta_{i} t}\right)^{1-x_{i}},
$$

by total state enumeration. This method is computationally inefficient, but will serve the purpose to prove Theorem 5.3 to follow. In applications more efficient approaches are needed such as for instance the technique of recursive disjoint products, see Abraham (1979), Ball \& Provan (1988) and Locks (1980, 1982). For network systems the factoring algorithm can be very efficient, see Satyanarayana \& Chang (1983).

The following lemma is obtained immediately from Theorem 2.1 using (5.4).

Lemma 5.2 For the case of exponentially distributed component lifetimes the likelihoood function in Theorem 2.1 can be written in the form

$$
L(\boldsymbol{\theta})=\sum_{k=1}^{K} \prod_{i \in B_{k}} f\left(\theta_{i} ; 1,0, t_{k, i}\right) \prod_{i \in C_{k}} f\left(\theta_{i} ; 1, t_{k, i}\right) \prod_{i \in D_{k}} f\left(\theta_{i} ; 2, t_{k, i}\right),
$$

where $B_{k}, C_{k}, D_{k}$ are disjoint subsets of $E$ for each $k=1, \ldots, K$.

Our main result in our Bayesian approach is the following theorem being completely parallel to Theorem 2.2 in Gåsemyr \& Natvig (1998).

Theorem 5.3 a) Suppose that the failure rates $\theta_{i}, i \in E$ for the components of a binary, monotone system $(E, \phi)$ have a joint prior distribution of the form

$$
\pi(\boldsymbol{\theta}) \propto \sum_{j=1}^{J} \prod_{i=1}^{n} f\left(\theta_{i} ; a_{j, i}, b_{j, i}, \boldsymbol{t}_{j, i}\right)=\sum_{j=1}^{J} \prod_{i=1}^{n} \gamma\left(a_{j, i}, b_{j, i}, \boldsymbol{t}_{j, i}\right)^{-1} g\left(\theta_{i} ; a_{j, i}, b_{j, i}, \boldsymbol{t}_{j, i}\right) .
$$

Then the posterior distribution of $\boldsymbol{\theta}$ associated with the likelihood function given by (5.5) is of the form

$$
\begin{aligned}
\pi(\boldsymbol{\theta} \mid \boldsymbol{D}) & \propto \sum_{j=1}^{J} \sum_{k=1}^{K}\left[\prod_{i \in B_{k}} f\left(\theta_{i} ; a_{j, i}, b_{j, i}, \boldsymbol{t}_{j, i}, t_{k, i}\right)\right] \\
\times & \left.\times \prod_{i \in C_{k}} f\left(\theta_{i} ; a_{j, i}, b_{j, i}+t_{k, i}, \boldsymbol{t}_{j, i}\right)\right] \prod_{i \in D_{k}} f\left(\theta_{i} ; a_{j, i}+1, b_{j, i}+t_{k, i}, \boldsymbol{t}_{j, i}\right) \\
& \times\left[\prod_{i \in E-\left(B_{k} \cup C_{k} \cup D_{k}\right)} f\left(\theta_{i} ; a_{j, i}, b_{j, i}, \boldsymbol{t}_{j, i}\right)\right] .
\end{aligned}
$$

b) The class of distributions of the form (5.6) is a conjugate class of priors for our exponential model. 
c) Suppose the prior distribution,

$$
\pi(\boldsymbol{\theta})=\prod_{i=1}^{n} g\left(\theta_{i} ; a_{i}, b_{i}\right)
$$

for $\boldsymbol{\theta}$ is updated with data from $r$ independent systems with likelihood functions of the form given by Theorem 2.1. Then the posterior distribution is of the form (5.6). If especially the likelihood functions are of the form given by Corollary 3.1, the posterior distribution reduces to a product of generalized gamma distributions.

Proof a) is a straightforward application of Bayes theorem. b) follows since (5.7) is of the same general form as (5.6). c) follows by repeated use of a) remembering Corollary 3.2.

Theorem 5.3 states that the weighted sum of products of generalized gamma distributions is the conjugate prior for $\underline{\theta}$ with respect to our exponential model. This seems to be a completely new generalization of the fact that the gamma distribution is the conjugate prior for the failure rate in an exponential model, parallel to the generalization given in Gåsemyr \& Natvig (1998). For further comments we refer to that paper and to Gåsemyr et al. (1999) and Sørensen (1999) where simulation techniques to arrive at the posterior distribution in the model of Gåsemyr \& Natvig (1998) are given.

By considering the likelihood functions given in Theorems 4.1 and 4.2 instead of the one given in Theorem 2.1, Theorem 5.3 is still valid. This follows since for the case of exponentially distributed component lifetimes we have

$$
\begin{aligned}
& F_{i}\left(\tau_{i, \ell}-S_{i, \ell-1}\right)-F_{i}\left(T^{R_{i, \ell}}-S_{i, \ell-1}\right)= \\
& \quad \exp \left(-\theta_{i}\left(T^{R_{i, \ell}}-S_{i, \ell-1}\right)\right)\left[1-\exp \left(-\theta_{i}\left(\tau_{i, \ell}-T^{R_{i, \ell}}\right)\right)\right] .
\end{aligned}
$$

We conclude this section by returning to Example 4.1.

Example 5.1 (example 4.1 continued) Assume that we choose the failure rates $\theta_{1}, \ldots, \theta_{7}$ to be independent à priori each having an ordinary gamma distribution $g(\theta ; 1,1000)$ with expectation and standard deviation equal to 0.001 . From Corollary 3.2 and (4.1) $\theta_{1}, \ldots, \theta_{7}$ are independent à posteriori as well. The posterior distribution of $\theta_{i}$ is $g\left(\theta_{i} ; a_{i}, b_{i}\right), i=1,2,3,4$ with $\left(a_{i}, b_{i}\right)$ respectively equal to $(12,10395),(4,11000),(6,10128)$ and $(6,11000)$. For $i=5,6,7$ the posterior distribution is $g\left(\theta_{i} ; a_{i}, b_{i}, \boldsymbol{t}_{i}\right)$ with $\left(a_{i}, b_{i}, \boldsymbol{t}_{i}\right)$ respectively equal to

$$
\begin{aligned}
& (3,5497,449,273,447,337,1614,860,1223,295), \\
& (2,3904,866,346,253,499,449,115,337,1614,1223,284,810,295) \\
& (2,4682,866,346,499,449,337,1614,1223,284,299,396)
\end{aligned}
$$

From (5.1) and (5.3) it follows that the expected value in the $g\left(\theta_{i} ; a_{i}, b_{i}, \boldsymbol{t}_{i}\right)$ distribution is $\gamma\left(a_{i}, b_{i}, \boldsymbol{t}_{i}\right) / \gamma\left(a_{i}+1, b_{i}, \boldsymbol{t}_{i}\right)$ whereas we know that it is $a_{i} / b_{i}$ in the $g\left(\theta_{i} ; a_{i}, b_{i}\right)$ distribution. In Table 4 the correct values of $\theta_{i}$ is compared with the posterior expected values, whereas the posterior for $\theta_{7}$ is plotted in Figure 2.

Table 4. Comparison of correct values of $\theta_{i}$ with posterior expectations

\begin{tabular}{l|c|c|c|c|c|c|c|}
\hline Component & 1 & 2 & 3 & 4 & 5 & 6 & 7 \\
\hline Correct $\theta_{i}$ & 0.001 & 0.0005 & 0.00056 & 0.00067 & 0.00167 & 0.00125 & 0.00143 \\
\hline Posterior expectations & 0.00115 & 0.00036 & 0.00059 & 0.00055 & 0.00145 & 0.00221 & 0.00171 \\
\hline
\end{tabular}


Figure 2. The posterior probability density function for $\theta_{7}$.

\section{Acknowledgement}

We are thankful to cand.scient. Erik Sørensen for writing a computer code providing the posterior expectations for components 5, 6 and 7 in Table 4 and the plot in Figure 2.

\section{References}

J.A. Abraham, "An improved algorithm for network reliability," IEEE Trans.Reliability vol. 28, pp. 58-61, 1979.

M.O. Ball and J.S. Provan, "Disjoint products and efficient computation of reliability," Operations Research vol. 36, pp. 703-715, 1988.

J.A. Bondy and U.S.R. Murty, Graph Theory with Applications, North Holland: New York, N.Y., 1976.

J. Gåsemyr, "On identifiability in the autopsy model of reliability systems of lifemonitored and conditionally lifemonitored components," J. Appl. Prob. vol. 35, pp. 427-437, 1998.

J. Gåsemyr and B. Natvig, "The posterior distribution of the parameters of component lifetimes based on autopsy data in a shock model," Scand. J. Statist. vol. 25, pp. 271292, 1998.

J. Gåsemyr, B. Natvig and E.K.M. Sørensen, "A comparison of two sequential MetropolisHastings algorithms with standard simulation techniques in Bayesian inference in reliability models involving the generalized gamma distribution," Statistical Research Report, Dept. of Mathematics, University of Oslo, 1999. 
M.O. Locks, "Recursive disjoint products, inclusion-exclusion, and min-cut approximations," IEEE. Trans. Reliability vol. 29, pp. 368-371, 1980.

M.O. Locks, "Recursive disjoint products: a review of three algorithms," IEEE. Trans. Reliability vol. 31, pp. 33-35, 1982.

I. Meilijson, "Estimation of the lifetime distribution of the parts from the autopsy statistics of the machine," J. Appl. Prob. vol. 18, pp. 829-838, 1981.

I. Meilijson, "Competing risks on coherent reliability systems: Estimation in the parametric case," J. Amer. Statist. Ass. vol. 89, pp. 1459-1464, 1994.

B. Natvig, "New light on measures of importance of system components," Scand. J. Statist. vol. 12, pp. 43-54, 1985.

A. Satyanarayana and M.K. Chang, "Network reliability and the factoring theorem," Networks vol. 13, pp. 107-120, 1983.

A.F.M. Smith and G.O. Roberts, "Bayesian computation via the Gibbs sampler and related Markov Chain Monte Carlo methods," J.R.Statist.Soc. B. vol. 55, pp. 3-23, 53-102, 1993.

E.K.M. Sørensen, "Simulating the posterior distribution in reliability models involving the generalized gamma distribution," Cand.scient. thesis, Dept. of Mathematics, University of Oslo, 1999. 


\section{Appendix 1 Proof of theorems}

Introduce

$$
R_{k}(t)=\bigcap_{\ell \in R_{k}}\left(T_{\ell}>t\right), \quad t>Z_{k} \quad N^{k}=\bigcup_{\ell=1}^{k} H_{\ell}
$$

Lemma A1.1 For $0 \leq k \leq K$ and $t>Z_{k}$ define

$$
\begin{aligned}
\rho_{i, \boldsymbol{y}, j}\left(t ; Z_{1}, I_{1}, \boldsymbol{Y}_{1}, \ldots, Z_{k}, I_{k}, \boldsymbol{Y}_{k}\right) \\
=\lim _{d t \rightarrow 0} P\left[\left(t<Z_{k+1} \leq t+d t\right) \cap\left(I_{k+1}=i\right)\right. \\
\left.\cap\left(\boldsymbol{Y}_{k+1}=\boldsymbol{y}\right) \cap\left(J_{k+1}=j\right) \mid \mathcal{B}_{k} \cap\left(Z_{k+1}>t\right)\right] / d t \\
=\lim _{d t \rightarrow 0} P\left[\left(t<Z_{k+1} \leq t+d t\right) \cap\left(I_{k+1}=i\right)\right. \\
\left.\cap\left(\boldsymbol{Y}_{k+1}=\boldsymbol{y}\right) \cap\left(J_{k+1}=j\right) \mid \mathcal{E}_{k} \cap R_{k}(t) \cap(T>t)\right] / d t .
\end{aligned}
$$

Then

i) for $i \in R_{k}, \boldsymbol{y} \in \mathcal{Y}_{k+1}$

$$
\begin{aligned}
& \rho_{i, \boldsymbol{y}, 0}\left(t ; Z_{1}, I_{1}, \boldsymbol{Y}_{1}, \ldots, Z_{k}, I_{k}, \boldsymbol{Y}_{k}\right) \\
& =\lambda_{i}(t) \prod_{\ell \in G_{0}(\boldsymbol{y})} F_{\ell}(t) \prod_{\ell \in G_{1}(\boldsymbol{y})} \bar{F}_{\ell}(t) h\left(\mathbf{1}_{\left(R_{k}-\{i\}\right) \cup G_{1}(\boldsymbol{y})}, \mathbf{0}_{Q_{k} \cup\{i\} \cup G_{0}(\boldsymbol{y})}, \overline{\boldsymbol{F}}(t)\right) / \\
& \quad h\left(\mathbf{1}_{R_{k}}, \mathbf{0}_{Q_{k}}, \overline{\boldsymbol{F}}(t)\right)
\end{aligned}
$$

ii) for $i \in R_{k} \cap C_{A_{j}}$ where $j$ is such that $Q_{k} \subseteq A_{j}, R_{k}-\{i\} \subseteq A_{j}^{c}$

$$
\begin{aligned}
& \rho_{i,-\mathbf{1}, j}\left(t, Z_{1}, I_{1}, \boldsymbol{Y}_{1}, \ldots, Z_{k}, I_{k}, \boldsymbol{Y}_{k}\right) \\
& \quad=\lambda_{i}(t) \prod_{\ell \in A_{j}-\left(M \cup N^{k}\right)} F_{\ell}(t) \prod_{\ell \in A_{j}^{c}-\left(M \cup N^{k}\right)} \bar{F}_{\ell}(t) / h\left(\mathbf{1}_{R_{k}}, \mathbf{0}_{Q_{k}}, \overline{\boldsymbol{F}}(t)\right)
\end{aligned}
$$

iii) for $j$ such that $R_{k} \subseteq A_{j}^{c}, Q_{k} \subseteq A_{j}, C_{A_{j}}-\left(M \cup N^{k}\right) \neq \emptyset$

$$
\begin{aligned}
& \rho_{0,-\mathbf{1}, j}\left(t ; Z_{1}, I_{1}, \boldsymbol{Y}_{1}, \ldots, Z_{k}, I_{k}, \boldsymbol{Y}_{k}\right) \\
& \sum_{i \in C_{A_{j}}-\left(M \cup N^{k}\right)} \lambda_{i}(t) \prod_{\ell \in A_{j}-\left(M \cup N^{k} \cup\{i\}\right)} F_{\ell}(t) \prod_{\ell \in\left(A_{j}^{c} \cup\{i\}\right)-\left(M \cup N^{k}\right)} \bar{F}_{\ell}(t) / h\left(\mathbf{1}_{R_{k}}, \mathbf{0}_{Q_{k}}, \overline{\boldsymbol{F}}(t)\right)
\end{aligned}
$$

iv) otherwise

$$
\rho_{i, \boldsymbol{y}, j}\left(t ; Z_{1}, I_{1}, \boldsymbol{Y}_{1}, \ldots, Z_{k}, I_{k}, \boldsymbol{Y}_{k}\right)=0
$$

Proof i) $\rho_{i, \boldsymbol{y}, 0}\left(t ; Z_{1}, I_{1}, \boldsymbol{Y}_{1}, \ldots, Z_{k}, I_{k}, \boldsymbol{Y}_{k}\right)$

$$
\begin{gathered}
=\lim _{d t \rightarrow 0} P\left[\left(t<T_{i} \leq t+d t\right) \cap\left(\bigcap_{\ell \in R_{k}-\{i\}}\left(T_{\ell}>T_{i}\right)\right) \cap\left(\boldsymbol{Y}_{k+1}^{*}=\boldsymbol{y}\right) \cap\left(T>T_{i}\right) \mid \mathcal{E}_{k} \cap R_{k}(t)\right] / \\
\quad\left\{P\left[T>t \mid \mathcal{E}_{k} \cap R_{k}(t)\right] d t\right\} \\
=\lambda_{i}(t) \lim _{d t \rightarrow 0}\left\{P\left[\bigcap_{\ell \in R_{k}-\{i\}}\left(T_{\ell}>T_{i}\right) \mid \mathcal{E}_{k} \cap R_{k}(t) \cap\left(t<T_{i} \leq t+d t\right)\right]\right.
\end{gathered}
$$




$$
\begin{aligned}
& \times P\left[\boldsymbol{Y}_{k+1}^{*}=\boldsymbol{y} \mid \mathcal{E}_{k} \cap\left(t<T_{i} \leq t+d t\right) \cap\left(\bigcap_{\ell \in R_{k}-\{i\}}\left(T_{\ell}>T_{i}\right)\right)\right] \\
& \left.\times P\left[T>T_{i} \mid \mathcal{E}_{k} \cap\left(t<T_{i} \leq t+d t\right) \cap\left(\bigcap_{\ell \in R_{k}-\{i\}}\left(T_{\ell}>T_{i}\right)\right) \cap\left(\boldsymbol{Y}_{k+1}^{*}=\boldsymbol{y}\right)\right]\right\} / \\
& \quad P\left[\phi\left(\mathbf{1}_{R_{k}}, \mathbf{0}_{Q_{k}}, \boldsymbol{X}(t)\right)=1\right]
\end{aligned}
$$

By applying (2.3) the proof is completed.

ii) $\rho_{i,-1, j}\left(t ; Z_{1}, I_{1}, \boldsymbol{Y}_{1}, \ldots, Z_{k}, I_{k}, \boldsymbol{Y}_{k}\right)$

$$
\begin{aligned}
&= \lim _{d t \rightarrow 0} P\left[\left(t<T_{i} \leq t+d t\right) \cap\left(\bigcap_{\ell \in R_{k}-\{i\}}\left(T_{\ell}>T_{i}\right)\right) \cap\left(\bigcap_{\ell \in A_{j}-\left(M \cup N^{k}\right)}\left(T_{\ell}<T_{i}\right)\right) \cap\right. \\
&\left.\left(\bigcap_{\ell \in A_{j}^{c}-\left(M \cup N^{k}\right)}\left(T_{\ell}>T_{i}\right)\right) \mid \mathcal{E}_{k} \cap R_{k}(t)\right] /\left\{P\left[T>t \mid \mathcal{E}_{k} \cap R_{k}(t)\right] d t\right\} \\
&=\lambda_{i}(t)\left\{\operatorname { l i m } _ { d t \rightarrow 0 } \left[P \left[\bigcap_{\ell \in R_{k}-\{i\}}\left(T_{\ell}>T_{i}\right) \cap\left(\bigcap_{\ell \in A_{j}-\left(M \cup N^{k}\right)}\left(T_{\ell}<T_{i}\right)\right) \cap\right.\right.\right. \\
&\left.\left.\quad\left(\bigcap_{\ell \in A_{j}^{c}-\left(M \cup N^{k}\right)}\left(T_{\ell}>T_{i}\right)\right) \mid \mathcal{E}_{k} \cap R_{k}(t) \cap\left(t<T_{i} \leq t+d t\right)\right]\right\} / h\left(\mathbf{1}_{R_{k}}, \mathbf{0}_{Q_{k}}, \overline{\boldsymbol{F}}(t)\right),
\end{aligned}
$$

completing the proof.

iii) $\rho_{0,-1, j}\left(t ; Z_{1}, I_{1}, \boldsymbol{Y}_{1}, \ldots, Z_{k}, I_{k}, \boldsymbol{Y}_{k}\right)$

$$
\begin{gathered}
=\lim _{d t \rightarrow 0} P\left[\bigcup _ { i \in C _ { A _ { j } } - ( M \cup N ^ { k } ) } \left\{\left(t<T_{i} \leq t+d t\right) \cap\left(\bigcap_{\ell \in R_{k}}\left(T_{\ell}>T_{i}\right)\right) \cap\left(\bigcap_{\ell \in A_{j}-\left(M \cup N^{k} \cup\{i\}\right)}\left(T_{\ell}<T_{i}\right)\right) \cap\right.\right. \\
\left.\left.\quad\left(\bigcap_{\ell \in A_{j}^{c}-\left(M \cup N^{k}\right)}\left(T_{\ell}>T_{i}\right)\right)\right\} \mid \mathcal{E}_{k} \cap R_{k}(t)\right] /\left\{P\left[T>t \mid \mathcal{E}_{k} \cap R_{k}(t)\right] d t\right\},
\end{gathered}
$$

completing the proof.

Proof of Theorem 2.1 For $0 \leq k \leq K$ and $t>Z_{k}$ we have

$$
\begin{aligned}
& P\left(Z_{k+1}>t \mid \mathcal{B}_{k}\right)=P\left[R_{k}(t) \cap(T>t) \mid \mathcal{E}_{k} \cap\left(T>Z_{k}\right)\right] \\
& \quad=P\left(R_{k}(t) \mid \mathcal{E}_{k}\right) P\left[T>t \mid \mathcal{E}_{k} \cap R_{k}(t)\right] / P\left(T>Z_{k} \mid \mathcal{E}_{k}\right) \\
& \quad=\prod_{\ell \in R_{k}}\left(\bar{F}_{\ell}(t) / \bar{F}_{\ell}\left(Z_{k}\right)\right) h\left(\mathbf{1}_{R_{k}}, \mathbf{0}_{Q_{k}}, \overline{\boldsymbol{F}}(t)\right) / h\left(\mathbf{1}_{R_{k}}, \mathbf{0}_{Q_{k}}, \overline{\boldsymbol{F}}\left(Z_{k}\right)\right)
\end{aligned}
$$

Inserting this in (2.2) with $k_{0}=K$, using case i) of Lemma A1.1 and (2.1) we obtain

$$
\begin{aligned}
& L\left(\boldsymbol{\theta} \mid \mathcal{B}_{K}\right)=\prod_{k=0}^{K-1}\left\{\prod_{\ell \in R_{k}}\left[\left(\bar{F}_{\ell}\left(Z_{k+1}\right) / \bar{F}_{\ell}\left(Z_{k}\right)\right) h\left(\mathbf{1}_{R_{k}}, \mathbf{0}_{Q_{k}}, \overline{\boldsymbol{F}}\left(Z_{k+1}\right)\right) / h\left(\mathbf{1}_{R_{k}}, \mathbf{0}_{Q_{k}}, \overline{\boldsymbol{F}}\left(Z_{k}\right)\right)\right]\right. \\
& \times \lambda_{I_{k+1}}\left(Z_{k+1}\right) \prod_{\ell \in H_{k+1,0}} F_{\ell}\left(Z_{k+1}\right) \prod_{\ell \in H_{k+1,1}} \bar{F}_{\ell}\left(Z_{k+1}\right) \\
& \left.h\left(\mathbf{1}_{\left(R_{k}-\left\{I_{k+1}\right\}\right) \cup H_{k+1,1}}, \mathbf{0}_{Q_{k} \cup\left\{I_{k+1}\right\} \cup H_{k+1,0}}, \overline{\boldsymbol{F}}\left(Z_{k+1}\right)\right) / h\left(\mathbf{1}_{R_{k}}, \mathbf{0}_{Q_{k}}, \overline{\boldsymbol{F}}\left(Z_{k+1}\right)\right)\right\} \\
& =\prod_{i \in N_{0}} F_{i}\left(\tau_{i}\right) \prod_{k=0}^{K-1}\left\{\left[\prod_{\ell \in R_{k+1} \cup\left\{I_{k+1}\right\}} \bar{F}_{\ell}\left(Z_{k+1}\right) / \prod_{\ell \in R_{k}} \bar{F}_{\ell}\left(Z_{k}\right)\right] \lambda_{I_{k+1}}\left(Z_{k+1}\right)\right.
\end{aligned}
$$




$$
\begin{aligned}
& \left.\times h\left(\mathbf{1}_{R_{k+1}}, \mathbf{0}_{Q_{k+1}}, \overline{\boldsymbol{F}}\left(Z_{k+1}\right)\right) / h\left(\mathbf{1}_{R_{k}}, \mathbf{0}_{Q_{k}}, \overline{\boldsymbol{F}}\left(Z_{k}\right)\right)\right\} \\
= & \prod_{i \in N_{0}} F_{i}\left(\tau_{i}\right) \prod_{\ell \in R_{K}} \bar{F}_{\ell}\left(Z_{K}\right) \prod_{k=0}^{K-1} \bar{F}_{I_{k+1}}\left(Z_{k+1}\right) \lambda_{I_{k+1}}\left(Z_{k+1}\right) h\left(\mathbf{1}_{R_{K}}, \mathbf{0}_{Q_{K}}, \overline{\boldsymbol{F}}\left(Z_{K}\right)\right)
\end{aligned}
$$

To obtain the full likelihood, we multiply this expression with the contribution from the interval $\left(Z_{K}, S\right]$, which is computed by another application of (A1.1) with $k$ replaced by $K$ and $t$ by $S$, and by either using case ii) or iii) of Lemma A1.1 when $V>S$.

Proof of Theorem 4.1 The likelihood is found by linking contributions from the consecutive operational periods of the system. With obvious notation we then have

$$
L(\boldsymbol{\theta})=L\left(\boldsymbol{\theta} \mid\left(0, t_{0}\right]\right)=\prod_{r=0}^{R-1}\left[L\left(\boldsymbol{\theta} \mid\left(T^{r}, T^{r+1}\right]\right)\right] L\left(\boldsymbol{\theta} \mid\left(T^{R}, t_{0}\right]\right) .
$$

The available information at the beginning of the $(r+1)$ th operational period is $\mathcal{B}_{k_{r}}$. Introduce the corresponding conditional distributions $(r=0,1, \ldots, R-1)$

$$
G_{i}^{r}(t)=P\left(X_{i, r+1}(t)=0 \mid \mathcal{B}_{k_{r}}\right), \quad t>T^{r},
$$

with corresponding p.d.f. $g_{i}^{r}(t)$ and failure rate $\gamma_{i}^{r}(t)=g_{i}^{r}(t) / \bar{G}_{i}^{r}(t), i \in E$.

Furthermore, introduce

$$
N_{0}^{r}=\bigcup_{k_{r}+1 \leq k \leq k_{r+1}} H_{k, 0}
$$

$=$ the set of components being failed on inspections in the $(r+1)$ th operational period

$$
\begin{aligned}
N_{0}^{R} & =\underset{\left\{k \geq k_{R}+1 \mid Z_{k}^{*}<t_{0}\right\}}{\bigcup} H_{k, 0} \\
& =\text { the set of components being failed on inspections in }\left(T^{R}, t_{0}\right)
\end{aligned}
$$

$$
Q^{r}=\bigcup_{k_{r}+1 \leq k \leq k_{r+1}}\left\{I_{k}^{*}\right\}
$$

$=$ the set of components observed to fail in the $(r+1)$ th operational period $Q^{R}=\bigcup_{\left\{k \geq k_{R}+1 \mid Z_{k}^{*}<t_{0}\right\}}\left\{I_{k}^{*}\right\}$

$=$ the set of components observed to fail in $\left(T^{R}, t_{0}\right)$

$$
A_{J^{r+1}}=\left\{i \in E \mid X_{i, r+1}\left(T^{r+1}\right)=0\right\}
$$

$=$ the fatal set corresponding to the $(r+1)$ th jump of $\psi(\boldsymbol{X}(t))$ to zero

$$
L_{i}^{r}=\min \left\{\ell \in\{1,2, \ldots\} \mid T^{r}<S_{i, \ell}\right\}
$$

$=$ the number of renewal cycles for the $i$ th component needed to just exceed $T^{r}$

By applying Corollary 3.1 for the case $V>S$ with respect to $\psi$ we get

$$
\begin{aligned}
& L\left(\boldsymbol{\theta} \mid\left(T^{r}, T^{r+1}\right]\right)=\prod_{i \in Q^{r}} \gamma_{i}^{r}\left(T_{i, L_{i}^{r}}\right) \prod_{i \in Q^{r}} \bar{G}_{i}^{r}\left(T_{i, L_{i}^{r}}\right) \\
& \quad \times \prod_{i \in N_{0}^{r}} G_{i}^{r}\left(\tau_{i, L_{i}^{r}}\right) \prod_{i \in A_{J^{r+1}}-\left(Q^{r} \cup N_{0}^{r}\right)} G_{i}^{r}\left(T^{r+1}\right) \prod_{i \in A_{J^{c}}^{r+1}} \bar{G}_{i}^{r}\left(T^{r+1}\right) .
\end{aligned}
$$


Now we have

$$
\bar{G}_{i}^{r}(t)=\bar{F}_{i}\left(t-S_{i, L_{i}^{r}-1}\right) / \bar{F}_{i}\left(T^{r}-S_{i, L_{i}^{r}-1}\right), \quad t>T^{r}
$$

Furthermore,

$$
\begin{aligned}
& \tau_{i, L_{i}^{r}}=T^{r+1}, \quad i \in A_{J^{r+1}}-Q^{r} \cup N_{0}^{r} . \\
& S_{i, L_{i}^{r}-1}=S_{i, L_{i}^{r+1}-1}, \quad i \in A_{J^{r+1}}^{c}
\end{aligned}
$$

Hence from (A1.3), noting that for $i \in A_{J^{r}}, S_{i, L_{i}^{r}-1}=T^{r}$, we get

$$
\begin{aligned}
& L\left(\boldsymbol{\theta} \mid\left(T^{r}, T^{r+1}\right]\right)=\prod_{i \in A_{J^{r}}}\left[\bar{F}_{i}\left(T^{r}-S_{i, L_{i}^{r}-1}\right)\right]^{-1} \\
& \quad \times \prod_{i \in Q^{r}} \lambda_{i}\left(T_{i, L_{i}^{r}}-S_{i, L_{i}^{r}-1}\right) \prod_{i \in Q^{r}} \bar{F}_{i}\left(T_{i, L_{i}^{r}}-S_{i, L_{i}^{r}-1}\right) \\
& \quad \times \prod_{i \in A_{J^{r+1}}-Q^{r}}\left[F_{i}\left(\tau_{i, L_{i}^{r}}-S_{i, L_{i}^{r}-1}\right)-F_{i}\left(T^{r}-S_{i, L_{i}^{r}-1}\right)\right] \\
& \quad \times \prod_{i \in A_{J^{r+1}}} \bar{F}_{i}\left(T^{r+1}-S_{i, L_{i}^{r+1}-1}\right) .
\end{aligned}
$$

Correspondingly, by applying Corollary 3.1 for the case $V=S$ we get

$$
\begin{aligned}
& L\left(\boldsymbol{\theta} \mid\left(T^{R}, t_{0}\right]\right)=\prod_{i \in A_{J}^{c} R}\left[\bar{F}_{i}\left(T^{R}-S_{i, L_{i}^{R}-1}\right)\right]^{-1} \\
& \quad \times \prod_{i \in Q^{R}} \lambda_{i}\left(T_{i, L_{i}^{R}}-S_{i, L_{i}^{R}-1}\right) \prod_{i \in Q^{R}} \bar{F}_{i}\left(T_{i, L_{i}^{R}}-S_{i, L_{i}^{R}-1}\right) \\
& \quad \times \prod_{i \in N_{0}^{R}}\left[F_{i}\left(\tau_{i, L_{i}^{R}}-S_{i, L_{i}^{R}-1}\right)-F_{i}\left(T^{R}-S_{i, L_{i}^{R}-1}\right)\right] \\
& \quad \times \prod_{i \in R\left(t_{0}\right)} \bar{F}_{i}\left(t_{0}-S_{i, L_{i}^{R}-1}\right) \prod_{i \in E-\left(Q^{R} \cup N_{0}^{R} \cup R\left(t_{0}\right)\right)} \bar{F}_{i}\left(T^{R}-S_{i, L_{i}^{R}-1}\right) .
\end{aligned}
$$

Inserting (A1.4) and (A1.5) into (A1.2), changing the order of multiplication, we get

$$
\begin{aligned}
& L(\boldsymbol{\theta})=\prod_{i \in E}\left\{\prod_{r=0}^{R}\left[\lambda_{i}\left(T_{i, L_{i}^{r}}-S_{i, L_{i}^{r}-1}\right) \bar{F}_{i}\left(T_{i, L_{i}^{r}}-S_{i, L_{i}^{r}-1}\right)\right]^{I\left(i \in Q^{r}\right)}\right. \\
& \times \prod_{r=0}^{R-1}\left[F_{i}\left(\tau_{i, L_{i}^{r}}-S_{i, L_{i}^{r}-1}\right)-F_{i}\left(T^{r}-S_{i, L_{i}^{r}-1}\right)\right]^{I\left(i \in A_{J^{r+1}}-Q^{r}\right)} \\
& \left.\times\left[F_{i}\left(\tau_{i, L_{i}^{R}}-S_{i, L_{i}^{R}-1}\right)-F_{i}\left(T^{R}-S_{i, L_{i}^{R}-1}\right)\right]^{I\left(i \in N_{0}^{R}\right)}\right\} \\
& \times \prod_{i \in R\left(t_{0}\right)} \bar{F}_{i}\left(t_{0}-S_{i, L_{i}^{R}-1}\right) \prod_{i \in E-\left(Q^{R} \cup N_{0}^{R} \cup R\left(t_{0}\right)\right)} \bar{F}_{i}\left(T^{R}-S_{i, L_{i}^{R}-1}\right) \\
& =\prod_{i \in E}\left\{\prod_{\ell=1}^{L_{i}}\left[\lambda_{i}\left(T_{i, \ell}-S_{i, \ell-1}\right) \bar{F}_{i}\left(T_{i, \ell}-S_{i, \ell-1}\right)\right]^{I\left(i \in Q^{R_{i, \ell}}\right)}\right. \\
& \left.\times\left[F_{i}\left(\tau_{i, \ell}-S_{i, \ell-1}\right)-F_{i}\left(T^{R_{i, \ell}}-S_{i, \ell-1}\right)\right]^{I\left(i \in A_{J^{R_{i, \ell}+1}}-Q^{R_{i, \ell}}\right) I\left(R^{i, \ell}<R\right)+I\left(i \in N_{0}^{R}\right) I\left(R^{i, \ell}=R\right)}\right\} \\
& \times \prod_{i \in R\left(t_{0}\right)} \bar{F}_{i}\left(t_{0}-S_{i, L_{i}^{R}-1}\right) \prod_{i \in E-\left(Q^{R} \cup N_{0}^{R} \cup R\left(t_{0}\right)\right)} \bar{F}_{i}\left(T^{R}-S_{i, L_{i}^{R}-1}\right),
\end{aligned}
$$


having used the fact that $L_{i}^{R_{i, \ell}}=\ell$ and that $L_{i}=L_{i}^{R}$ for $i \in Q^{R} \cup N_{0}^{R}$. By noting that $I\left(i \in Q^{R_{i, \ell}}\right)=\delta_{i, \ell}$ and $I\left(i \in A_{J^{R_{i, \ell}+1}}-Q^{R_{i, \ell}}\right) I\left(R^{i, \ell}<R\right)+I\left(i \in N_{0}^{R}\right) I\left(R^{i, \ell}=R\right)=1-\delta_{i, \ell}$ and finally that $L_{i}=L_{i}^{R}-1$ for $i \in E-\left(Q^{R} \cup N_{0}^{R}\right)$, our proof is completed.

Proof of Theorem 4.2 The proof is very similar to the one of Theorem 4.1. We now have

$$
\bar{G}_{i}^{r}(t)= \begin{cases}\bar{F}_{i}\left(t-S_{i, L_{i}^{r}-1}\right) / \bar{F}_{i}\left(T^{r}-S_{i, L_{i}^{r}-1}\right), & i \in R_{k_{r}-1}-I_{k_{r}}^{*}, \quad t>T^{r} \\ \bar{F}_{i}\left(t-S_{i, L_{i}^{r}-1}\right), & i \in M \cup C-\left(R_{k_{r}-1}-I_{k_{r}}^{*}\right), \quad t>T^{r}\end{cases}
$$

Hence, we get, noting that $N_{0}^{r} \subseteq M \cup C-\left(R_{k_{r}-1}-I_{k_{r}}^{*}\right)$

$$
\begin{aligned}
& L\left(\boldsymbol{\theta} \mid\left(T^{r}, T^{r+1}\right]\right)=\prod_{i \in R_{k_{r}-1}-I_{k_{r}}^{*}}\left[\bar{F}_{i}\left(T^{r}-S_{i, L_{i}^{r}-1}\right)\right]^{-1} \prod_{i \in Q^{r}} \lambda_{i}\left(T_{i, L_{i}^{r}}-S_{i, L_{i}^{r}-1}\right) \\
& \quad \times \prod_{i \in Q^{r}} \bar{F}_{i}\left(T_{i, L_{i}^{r}}-S_{i, L_{i}^{r}-1}\right) \prod_{i \in N_{0}^{r}} F_{i}\left(\tau_{i, L_{i}^{r}}-S_{i, L_{i}^{r}-1}\right) \prod_{i \in R_{k_{r+1}-1}-I_{k_{r+1}^{*}}^{*}} \bar{F}_{i}\left(T^{r+1}-S_{i, L_{i}^{r+1}-1}\right) \\
& L\left(\boldsymbol{\theta} \mid\left(T^{R}, t_{0}\right]\right)=\prod_{i \in R_{k_{R-1}}-I_{k_{R}}^{*}}\left[\bar{F}_{i}\left(T^{R}-S_{i, L_{i}^{R}-1}\right)\right]^{-1} \prod_{i \in Q^{R}} \lambda_{i}\left(T_{i, L_{i}^{R}}-S_{i, L_{i}^{R}-1}\right) \\
& \quad \times \prod_{i \in Q^{R}} \bar{F}_{i}\left(T_{i, L_{i}^{R}}-S_{i, L_{i}^{R}-1}\right) \prod_{i \in N_{0}^{R}} F_{i}\left(\tau_{i, L_{i}^{R}}-S_{i, L_{i}^{R}-1}\right) \prod_{i \in R\left(t_{0}\right)} \bar{F}_{i}\left(t_{0}-S_{i, L_{i}^{R}-1}\right),
\end{aligned}
$$

leading to the completion of the proof.

\section{Appendix 2 Remarks on inspection strategies}

We start by proving that condition (2.3) is satisfied, as claimed in Section 3, for two inspection strategies considered there. We start by (3.1). Note that for any $t>Z_{k}^{*}$ and $i \in R_{k}$ by the piecewise constancy of $H_{i}^{\mathcal{E}_{k}}(\cdot)$ there exists $d t>0$ such that $H_{i}^{\mathcal{E}_{k}}(s)$ is constant for $s \in(t, t+d t)$. This constant value equals $H_{k+1}$ by $(3.1)$ if $Z_{k+1}^{*} \in(t, t+d t)$ and $I_{k+1}^{*}=i$. Hence $\boldsymbol{y} \in \mathcal{Y}_{k+1}$ implies $G(\boldsymbol{y})=H_{k+1}$. We then have for $\boldsymbol{y} \in \mathcal{Y}_{k+1}$

$$
\begin{array}{rl}
\lim _{d t \rightarrow 0} & P\left[\boldsymbol{Y}_{k+1}^{*}=\boldsymbol{y} \mid \mathcal{E}_{k} \cap\left(t<Z_{k+1}^{*} \leq t+d t\right) \cap\left(I_{k+1}^{*}=i\right)\right] \\
= & \lim _{d t \rightarrow 0} P\left[\bigcap_{\ell \in G_{0}(\boldsymbol{y})}\left(T_{\ell}<T_{i}\right) \cap\left(\bigcap_{\ell \in G_{1}(\boldsymbol{y})}\left(T_{\ell}>T_{i}\right)\right) \mid\right. \\
& \left.\mathcal{E}_{k} \cap\left(t<T_{i} \leq t+d t\right) \cap\left(\bigcap_{\ell \in R_{k}-\{i\}}\left(T_{\ell}>T_{i}\right)\right)\right] \\
= & \prod_{\ell \in G_{0}(\boldsymbol{y})} F_{\ell}(t) \prod_{\ell \in G_{1}(\boldsymbol{y})} \bar{F}_{\ell}(t),
\end{array}
$$

remembering the note just after (2.3). Hence the proof is completed.

Now turn to the more flexible strategy where $H_{k+1}$ is allowed to depend more extensively on information that becomes available at $Z_{k+1}^{*}$. Again note that for any $t>Z_{k}^{*}$ and $i \in R_{k}$ there exists $d t>0$ such that $H_{i}^{\mathcal{E}_{k}}(s, R, Q)$ is constant for $s \in(t, t+d t)$ for each of the finitely many possible pairs $(R, Q)$. Hence, by an argument similar to the one used for (3.1), a single iteration where, say component $\ell$ is selected, contributes with a factor $\left(F_{\ell}(t)\right)^{1-y_{\ell}}\left(\bar{F}_{\ell}(t)\right)^{y_{\ell}}$. By multiplying these factors for $\ell \in G(\boldsymbol{y})$ we get $(2.3)$. 
Next consider the assumption that inspections take zero operational time. This assumption can be justified if the operation of the system is interrupted during the inspections and time is taken as operational time, or if the inspections actually take a negligible amount of time compared to the lifetimes of the components. In the following example we illustrate the possible consequences for (2.3) and Theorem 2.1 of taking into account the times it takes to inspect each component.

Example A2.1 Let $(E, \phi)$ be a 2-out-of-6 system with $M=\{1\}$ and $C=\{2,3,4,5\}$. Let $\varepsilon_{i}$ be the time it takes to inspect component $i, i=2, \ldots, 5$. We construct an inspection strategy which basically corresponds to an inspection plan of type 2 defined by an inspection function $H$. However, now new inspection decisions are taken consecutively by means of $H$ as the inspections of the individual components are completed. $H$ is partially specified through the equations $H(\emptyset,\{1\})=\{2,3\}, H(\emptyset,\{1,2\})=\{4\}, H(\emptyset,\{1,3\})=\{5\}$, $H(\emptyset,\{1,2,3\})=\{4\}, H(\{4\}, Q)=\emptyset$ if $Q=\{1,2\}$ or $\{1,2,3\}, H(\{5\}, Q)=\emptyset$ if $Q=\{1,3\}$ or $\{1,2,3\}$. If now $\max \left\{T_{2}, T_{3}\right\}<T_{1}, \min \left\{T_{4}, T_{5}\right\}>T_{1}+\max \left\{\varepsilon_{2}, \varepsilon_{3}\right\}$ it follows that the result of the inspection after $T_{1}$ depends on whether $\varepsilon_{2}<\varepsilon_{3}$ or vice versa, and hence (2.3) is not satisfied. Indeed, in the first case $H_{1}=\{2,3,4\}$ and $\boldsymbol{Y}_{1}^{*}=(0,0,1,-1)$ whereas in the second case $H_{1}=\{2,3,5\}$ and $\boldsymbol{Y}_{1}^{*}=(0,0,-1,1)$. Of course, an ordinary inspection plan of type 2 is obtained if one instead waits until $T_{1}+\max \left\{\varepsilon_{2}, \varepsilon_{3}\right\}$ before a new decision is made, now resulting in $H_{1}=\{2,3,4\}$ and $\boldsymbol{Y}_{1}^{*}=(0,0,1,-1)$.

If the probability that $\varepsilon_{2}<\varepsilon_{3}$ does depend on component lifetimes or $\boldsymbol{\theta}$, which for instance would be the case if it takes less time to inspect a failed than a functioning component, then Theorem 2.1 is no longer valid. 